\title{
Scientific Perspectivism and the Methodology of Modern Mathematical Physics
}

\author{
Noah Stemeroff ${ }^{1,2}$ (1) \\ ${ }^{1}$ Lichtenberg Group for the History and Philosophy of Physics, University of Bonn, Bonn, Germany and ${ }^{2}$ Cohn \\ Institute for the History and Philosophy of Science and Ideas, Tel Aviv University, Tel Aviv-Yafo, Israel \\ Emails: nstemero@uni-bonn.de; ns1@mail.tau.ac.il
}

(Received 19 January 2021; revised 21 April 2021; accepted 10 May 2021; first published online 12 January 2022)

\begin{abstract}
Perspectival realists often appeal to the methodology of science to secure a realist account of the retention and continued success of scientific claims through the progress of science (e.g., Massimi [2016]). However, in the context of modern physics, the retention and continued success of scientific claims are typically only definable within a mathematical framework. In this article, I argue that this concern leaves the perspectivist open to Cassirer's (1910/ 1923) neo-Kantian critique of the applicability of mathematics in the natural sciences. To support this criticism, I present a case study on the conservation of energy in modern physics.
\end{abstract}

\section{Introduction}

Giere's (2006) Scientific Perspectivism seeks a middle ground between a historically motivated relativism and traditional scientific realism. He grants to the relativist that the history of science has shown that all scientific knowledge is contextual-in the sense that it is situated within a scientific framework. And given the contextual nature of scientific knowledge, Giere calls into question the traditional realist appeal to both objectivity and truth. But what makes Giere's perspectivism distinctive is its espousal as a form of scientific realism. ${ }^{1}$ He maintains that his perspectivism offers a sense in which modern science can still be said to say something about the natural world - that is, that according to a given successful theory, the world appears "to be roughly such and such" $(2006,6)$. This, he suggests, may be enough to support a genuine scientific realism.

\footnotetext{
${ }^{1}$ It is this realist claim that distinguishes Giere's perspectivism from its influential historical precedent in the philosophy of science. We can find antecedents to this view in the writings of Nietzsche (1873/2010), Cassirer (1910/1923), Carnap (1956), Kuhn (1962), and Goodman (1976, 1978).

(c) The Author(s), 2022. Published by Cambridge University Press on behalf of the Philosophy of Science Association. This is an Open Access article, distributed under the terms of the Creative Commons Attribution licence (http:// creativecommons.org/licenses/by/4.0/), which permits unrestricted re-use, distribution and reproduction, provided the original article is properly cited.
} 
However, lingering concerns remain as to whether Giere's perspectivism can make good on this realist claim. ${ }^{2}$ Giere denies the existence of a "God's-eye point of view" form of objectivity - that is, a complete and true description of the way the world is. ${ }^{3}$ The "world" that is represented in Giere's scientific perspectivism is not the traditional realist's world of an objective, mind-independent reality. Rather, it is a world constituted by a given scientific vantage point, beyond which no knowledge is taken to be possible. ${ }^{4}$ Thus, it seems that Giere's perspectivism may be fundamentally at odds with the central dogma of scientific realism - that is, to at least say something about the features of a mind-independent reality.

In light of these concerns, there have been a number of recent attempts to develop a new form of scientific perspectivism, one that can make good on a realist commitment (e.g., Teller 2011, 2012, 2019; Rueger 2016; Massimi 2012, 2016, 2018a, 2018c). Most prominent among these views is Massimi's (2012, 2016, 2018a) perspectivism, which seeks to defend a traditional realist account of truth-a "perspectival truth" concerning a mind-independent reality. To motivate this account, Massimi draws from both the Kantian tradition and recent work on the perspectival nature of scientific modeling (e.g., Rueger 2005; Teller 2011, 2012). She appeals to Kant to defend an epistemic pluralism but draws on the perspectival modeling literature to develop a correspondence theory of truth that supports this pluralism. Here, Massimi highlights the retention and continued success of certain scientific claims through the progress of science to ground an account of perspectival truth. It is this appeal to the methodology of science that secures a sense in which perspectival claims can transcend the bounds of mere perspective.

In this article, I will argue that there is an inherent tension in this new form of perspectivism, one that is rooted in the appeal to the methodology of science to defend an account of scientific knowledge. In the first section, I will briefly outline the essential features of Massimi's perspectivism. I will show that the perspectivist appeal to the methodology of science assumes that this methodology is truth-conducive and that it can secure a consistent and well-defined body of knowledge to which a perspectivist can lend support. In the second section, I will present a critique of the perspectivist appeal to the methodology of science in the context of modern physics - a critique that originates in Cassirer's (1910/1923) neo-Kantian attack on the realist account of scientific knowledge. In modern physics, I will argue that the retention and continued success of scientific claims, through the perspectival series of scientific theories, can only be defined within a given mathematical framework. However, the perspectivist faces a challenge in defining the sense in which this framework supports a realist account of truth. In the third section, I will provide a case study of a novel perspectival account of conservation laws in modern physics to further motivate this Kantian critique. This account will trace the formal grounding of global conservation laws in the transition from static spacetime theories, such as classical mechanics, to dynamical

\footnotetext{
${ }^{2}$ Here, I take scientific realism to be defined by the belief that mature and successful scientific theories provide a true, or approximately true, description of at least some aspects of both the observable and unobservable features of the natural world. Following Chakravartty (2007, 9), I will define scientific realism to entail three claims: (1) a metaphysical tenet-that is, that there exists a mind-independent reality; (2) a semantic tenet-that is, that scientific claims can be construed literally; and (3) an epistemic tenet - that is, that some scientific claims about the world are true (at least approximately).

${ }^{3}$ In this sense, Giere's perspectivism $(2006,6)$ echoes Putnam's $(1981,1987)$ internal realism.

${ }^{4}$ Giere's perspectivism is categorical, applying at all levels of scientific practice.
} 
spacetime theories, such as general relativity. It will highlight the fundamental role that mathematics plays in securing the retention and continued success of scientific claims through the progress of modern theoretical physics. In the fourth section, I consider whether Massimi's perspectival realist can offer a viable response to this neo-Kantian critique, and the extent to which this concern poses a challenge to other forms of perspectivism (e.g., Teller 2011, 2012, 2019). To conclude, I will consider what lessons we can draw from this case study.

\section{Massimi's scientific perspectivism}

Massimi's perspectivism seeks to at least say something "about facts not being shaped by scientific perspectives or truth relativized to them" (2018a, 170). In this context, Massimi draws an important distinction between two concepts that she suggests are often conflated in discussions on scientific realism-namely, objectivity and truth. She suggests that "one can accept and fully endorse that scientific inquiry is indeed pluralistic and that there is no unique, objective, and privileged epistemic vantage point without necessarily having to conclude that perspectives shape scientific facts or relativize truth" (2018a, 170). Here, Massimi (2018c) draws on the work of Rueger (2005, 2016), Teller (2011, 2012), and Morrison (2011) concerning the perspectival nature of scientific modeling. Teller $(2011,2012)$ has argued that since all scientific representation is imprecise and idealized, it may be possible to maintain a number of representations of reality without giving up on scientific realism. Teller $(2012,272)$ suggests that

science — and human knowledge generally_ [gives us] a vast plurality of representative tools, none of which works with both complete precision and accuracy. But a great many of the resulting representations, like not perfectly focused photographs, do give us real cognitive grip on real things.

Similarly, Massimi (2018c, 338) notes that the "realist quest can be vindicated when one considers the indispensable role that such a plurality of perspectival models plays in advancing our knowledge of what might be real." In highlighting the exploratory function of perspectival models, Massimi's perspectival realist can accept a form of epistemic pluralism and abandon the claim of a God's-eye-view objectivity because it is this concession that might allow the perspectivist to account for the situated nature of scientific knowledge. However, this does not mean that we have to give up on truth altogether.

All the perspectival realist needs is an account of scientific truth that is independent of any appeal to objectivity, and this is exactly what Massimi (2016) has put forward. At the outset, it is clear that scientific perspectives provide contextual truth conditions, but these truth conditions only support a notion of scientific truth that is contextualized within the limits afforded by a given scientific theory, either past or present $(2016,12)$. These conditions do not license an interperspectival truth, which would be required for a genuine correspondence claim. However, Massimi (2016,13-15) suggests that if these contextual truth conditions are accessible across scientific perspectives, then we could characterize a perspectival truth qua correspondence, contextualized but secured by a form of resilience and interperspective translatability. It is this continuity across perspectives that allows the 
perspectivist to determine whether a given scientific claim meets its original truth conditions from the perspective of our currently accepted theories. In fact, this notion of perspectival truth may be enough for the perspectival realist to make good on many of the typical realist commitments. Massimi's perspectival realist already supports the metaphysical tenet of scientific realism, as they accept the existence of a mind-independent reality, and the semantic tenet, as they construe scientific claims literally. This appeal to perspectival truth allows the perspectival realist to also accept the epistemic tenet, as they hold that in accepting a theoretical claim, they believe it to be true- understood as perspectival truth across theoretical frameworks (Massimi 2018a, 170-71).

The initial problem with Massimi's perspectivism is that it is not exactly clear how one can defend a genuine scientific realism simply as a result of these contextual truth conditions and their accessibility across scientific perspectives. Massimi (2016, 13-14) suggests that we should understand contextual truth conditions as standards of performance adequacy by which scientific claims are said to meet certain methodological constraints that define the successful application of a theory. In this case, the overarching methodology of science can serve to ground a notion of truth across perspectives. However, this may not be enough. As Rueger $(2016,402)$ has noted in the context of perspectival models,

[d]rawing on the analogy with different spatial perspectives onto the same object, the perspectival realist suggests that the seemingly incompatible representations given by our models are in fact just representations of the same (independently existing) target system from different perspectives or points of view.

To secure a viable realist notion of truth, it seems Massimi must show that the set of claims that a perspectival realist holds to be true within a scientific perspective are reconcilable, in the sense that they can be shown to be consistent with the existence of a unique mind-independent reality.

In response to this type of concern, Massimi can appeal to her notion of scientific progress. Massimi $(2018 \mathrm{a}, 172)$ suggests that

[s]cientific progress can be characterized in perspectivalist terms if we take scientific perspectives not just as contexts of use-laying down specific standards of performance adequacy for knowledge claims - but also, and most importantly, as contexts of assessment offering standpoints from which knowledge claims of other (past) scientific perspectives can be evaluated (in terms of their ongoing performance adequacy as set out by their original standards). Thus, scientific claims of our historical predecessors can be retained or withdrawn, depending on whether they continue to satisfy their original standards of performance-adequacy when assessed from another (subsequent) perspective.

Assuming that the progress of science leads toward a consistent theory-that is, those claims that are eventually retained are consistent with the existence of a unique mind-independent reality - then what results from this notion of scientific progress appears to be a more modest form of scientific realism. It is a perspectival realism 
that replaces the traditional realist notion of truth qua correspondence with an objective reality with truth qua correspondence established as a form of resilience and coherence across the progress of science. This is simply the best we can do within the framework of our currently successful theories-which are liable to change.

Massimi's perspectivism offers the hope of charting a middle path between a historically motivated relativism and traditional scientific realism. The shared methodology of modern science can secure the retention and continued success of scientific claims. So long as this methodology remains relatively stable through the progress of science, this humble realism may offer the promise of supporting a robust and consistent body of knowledge to which a perspectival realist can lend support. Some may still wonder whether this notion of perspectival truth can truly support a genuine scientific realism, but there is no question that it offers an interesting new perspective on the realism debate-one that may be more in line with the discordant nature of modern science. However, in the next section, I argue that there is an inherent tension in the perspectivist appeal to the methodology of theoretical physics to secure a form of scientific realism.

\section{Perspectivism and the methodology of modern physics}

Massimi assumes that the methods of modern science are truth-conducive-that is, they are able to secure a perspectival knowledge of the objects, structures, processes, and so forth that exist in nature, independent of any physical theory. This reification of the methodology of modern science, over and above its instrumental value, entails not only that these methods can discover truths about both the observable and unobservable world but also that this has, in fact, been achieved in the cases that support perspectival realism.

The initial concern is that it is not entirely clear whether a perspectivist can give a compelling argument to support this reification of the methods of modern sciencethat is, one that does not transgress upon the central dogma of perspectivism. At the very least, Massimi needs to clarify what it is about the methodology of science that allows us to suggest that the retained truths in the perspectival series of scientific theories have some grip on reality.

Consider Massimi's (2018a, 171) example of the contrasting properties assigned to water in fluid dynamics and statistical mechanics. ${ }^{5}$ In this example, it seems that we can determine whether the claim that water has viscosity is true or not. From the perspective of statistical mechanics, we can appeal to the molecular mean flow to secure a sense in which the claim that water has viscosity can be considered to be true within the context of fluid dynamics. However, this only works because fluid dynamics and statistical mechanics share a theoretical and mathematical framework. It is only through this shared framework that one can define what viscosity is from the perspective of statistical mechanics and show that it is consistent with the account of viscosity in fluid dynamics. Under the assumption that this accessibility persists in the perspectival series of scientific theories, then this shared framework can determine whether it is, in fact, true that water possesses viscosity. It is this condition - that is, that the shared framework of the two theories is retained through

\footnotetext{
${ }^{5}$ Massimi cites this example of Teller $(2011,2012)$ in defense of her notion of perspectival truth.
} 
the progress of science-that underwrites the correspondence claim. But this only works to the extent that the methodological framework of classical mechanics is assumed to be truth-conducive.

However, this reification of the methodology of science may be problematic in the context of modern physics. Here, the retention and continued success of scientific claims underwriting perspectival realism are often only definable within a shared mathematical framework. But in what sense can this framework be said to have any grip on reality to support the perspectival correspondence claim?

This concern has an influential precedent in the history of philosophy. Those familiar with the work of Cassirer (e.g., 1910/1923, 1925/1946, 1936/1956) will have already noted the parallels between Massimi's appeal to the methodology of science to secure a perspectival realism and a similar argument made by Cassirer to support a neo-Kantian account of the objectivity of science. Cassirer's neo-Kantian philosophy of science emphasized the constitutive role that mathematics plays in scientific thought, and he often sought to challenge the viability of a scientific realism grounded on the mathematical representation of nature. His general concern was that the mathematical formalism of modern physical theory, as a symbolism, "harbours the curse of mediacy" (1925/1946, 7). In Cassirer's view, what is symbolized in abstract mathematics cannot be an exact copy of what exists, and it seems that scientific knowledge, "whose task is to describe the real and lay bare its finest threads, begins by turning aside from this very reality and substituting for it the symbols of number and magnitude" (1910/1923, 117). The solution, as Cassirer saw it, was to simply accept the fundamental mathematical constitution of the scientific conception of the natural world and give up the naive realist claim of representing a mindindependent reality.

Cassirer held that the mathematization of nature serves as a necessary presupposition of scientific thought, but he did not believe that this, in any way, constrained the choice of mathematical formalism in modern physical theory. It is here that Cassirer, in line with the Marburg neo-Kantian tradition, took a step beyond Kant in allowing for the revision of the constitutive framework of scientific thought. However, in allowing for this revision, Cassirer was forced to face the specter of a pervasive relativism. Thus, Cassirer $(1910 / 1923,282)$ held that at the heart of mathematical physics lies the "riddle of knowledge." In response to this riddle, Cassirer $(1910 / 1923,321)$ argued that the relativist abolition of the standard of objectivity does not entail the abolition of the difference in the value and performance of various scientific theories. Scientific theories do not stand apart in their relation to the world, to be judged solely on their own merits, but rather as part of a progressive series. Cassirer suggested that it is this series, and the hopeful convergence of the theoretical framework of modern physical theory, that can take the place of an objective external reality. He argued that we "need, not the objectivity of absolute things, but rather the objective determinateness of the method of experience" (1910/1923, 322). This method entails a systematic progression toward ever-more-general mathematical formulations of the "fixed and permanent realm of objectively necessary relations" to which all thought is directed $(1910 / 1923,315)$. This formed the basis for his appeal to a brand of neo-Kantian structuralism (e.g., see Cassirer 1936/1956).

The basic disagreement between Massimi's perspectivism and Cassirer's neoKantianism is clear. Although they would both maintain that scientific knowledge 
is historically and culturally situated, Cassirer holds that no knowledge is possible outside the constitutive framework of science. In contrast, Massimi holds that such knowledge is possible, at least in principle. In defense of this view, Massimi appeals to the retention and continued success of certain scientific claims through the progress of science to secure a sense in which they can be said to have some grip on reality. However, Cassirer would likely complain that the perspectivist's realism seems to remain forever in the shadows. The interperspective accessibility of scientific claims secured within the framework of the methodology guiding scientific progress does not, on its own, entail that the claims that a perspectivist accepts correspond to the world, as opposed to the constitutive framework that Cassirer would argue underwrites scientific progress.

In response to this Kantian concern, the perspectivist needs to specify the sense in which the methodological framework of modern science is able to secure a viable perspectival realism. Massimi (2018a, 171) suggests that perspectivism shares "with scientific realism the view that worldly states of affairs, the language of science, and truth as correspondence with states of affairs are all perspective independent." But what supports this correspondence claim? The perspectival account of truth is grounded on the continued success of scientific claims, defined in terms of Massimi's appeal to standards of performance adequacy, which she takes to be common across perspectives. These standards include empirical testability, projectability, fruitfulness, and so forth (Massimi 2016, 14). But in modern physics, these standards are often only definable within a mathematical framework. Thus, the perspectivist is required, at least in the context of modern physics, to give a response to the neo-Kantian critique of the applicability of mathematics in science. To further clarify this concern, I will now turn to a novel perspectivist case study concerning the conservation of energy in static and dynamical spacetime theories.

\section{A perspectival account of energy conservation}

The principle of the conversation of energy stands as one of the pillars of modern science. It underwrites large portions of classical physics and is, to a large extent, upheld in one manner or another in almost all branches of modern physics. Thus, the perspectival realist might want to suggest that the principle of the conservation of energy is true (e.g., see Massimi 2018b). The intertranslatability of the principle across perspectives, its adherence to the standards of performance adequacy, and its retention through the progress of science clearly support the perspectival truth claim. However, I will argue that the retention of energy-conservation laws in a dynamical spacetime theory, such as general relativity, can only be defined within the mathematical formalism of modern differential geometry.

In modern physics, the law of the conservation of energy is typically presented as a canonical application of the so-called "symmetry principle." This principle states that symmetries lie at the heart of modern physics. In particular, it suggests that the dynamical laws that govern any system should be derivable from the fundamental symmetries and invariances of a physical theory. The significance of the concepts of symmetry and invariance in modern physical theory concerns the way in which we typically represent the physical structure of the natural world. Following Weyl (1952, 7-8), it has become natural to suggest that "the mathematical laws governing 
nature are the origin of symmetry in nature." Indeed, Noether's theorem is thought to formally establish this connection in theories that can be given a Lagrangian formulation. But what is the mathematical conception of symmetry engendered by these laws?

To gain a better understanding of the mathematical concepts of symmetry and invariance, we can start by exploring a simple case: that of bilateral symmetry. In Weyl's influential definition $(1952,17-18)$, a body is defined to be bilaterally symmetric with respect to a given plane if it is carried onto itself by reflection in the plane. Reflection is defined mathematically through the notion of a mapping. A "mapping is defined whenever a rule is established by which every point $p$ is associated with an image $p_{0}$ " (Weyl 1952, 5). The reflection map, $S: p \rightarrow p_{0}$, carries an arbitrary point $p$ into its mirror image $p_{0}$. The same basic idea can be extended to characterize a rotational or translational symmetry. In each of these cases, the transformations that define the symmetries of the structure are automorphisms that map a structure onto itself. These transformations form a group, and the group describes the symmetry possessed by the structure (Weyl 1952, 45).

The geometrical connection between symmetry, invariance, and natural law in modern physical theory is based on the idea that the structure of the natural world can be taken to constitute a mathematical manifold. Any space can be taken to constitute a manifold so long as an open neighborhood of each point of the space has a continuous one-to-one mapping to an open neighborhood of $\Re^{n}$, the $n$-dimensional space of real numbers. In modern physics, we also typically demand that a manifold be differentiable. The differentiability of a manifold equips it with an enormous amount of additional structure, and this structure is fundamental to modern physical theory. As Schutz $(1980,23)$ notes: "It is hard to imagine a physical problem which does not involve some sort of continuous space." He points out that the "key to differential geometry's importance to modern physics is that it studies precisely those properties common to all such spaces." The differentiability of a manifold allows us to define dynamical equations and continuous symmetries. The continuous symmetries that play an especially important role within modern physical theory are the transformations that map dynamical equations and spacetime structures onto themselves. These automorphisms define the invariances that underwrite the conservation laws of modern physics.

The spacetime structures of classical physics, quantum theory, and special relativity are either Euclidean or pseudo-Euclidean. In each theory, if a system is subject to Hamilton's principle (which states that the motion of a system from time $t_{1}$ to $t_{2}$ is such that the integral $I=\int_{t_{1}}^{t_{2}} L d t$, has a stationary value for the actual path), then it is well known that the symmetries of the Lagrangian correspond to the existence of conserved quantities. Here, the integral $I$ is known as the action integral; $L$ is the Lagrangian and is defined as $L=T-V$, where $T$ is the kinetic energy, and $V$ is the potential energy of the system. In particular, it is easy to show that if the Lagrangian does not explicitly contain a particular coordinate dependence on the coordinate of displacement, then the corresponding canonical momentum is conserved. Of course, the same can be said for a coordinate of time and the conservation of energy. As Goldstein $(1980,588)$ notes, the "absence of explicit dependence on the coordinate means that the Lagrangian is unaffected by a transformation that alters the value of that coordinate; it is said to be invariant, or symmetric under the 
given transformation." It is clear why this is called a symmetry in the Lagrangian. The transformations of space or time map the Lagrangian onto itself. They are automorphisms that leave the structure unchanged.

Noether's first theorem generalizes this result and demonstrates that every continuous symmetry in the Lagrangian corresponds to a conserved quantity. ${ }^{6}$ However, to ensure the invariance of the Lagrangian, the continuous symmetry must correspond to a symmetry in the underlying metrical structure (Schutz 1980, 89). The reason why this must be the case is easy to see. If the definition of symmetry requires that an object or structure be mapped onto itself, then there must be a consistent means of defining the relevant notion of sameness. The motion must clearly take place along a continuous isometry in the spacetime-because it is the concept of an isometry that allows us to define the relevant notion of sameness (or congruence). ${ }^{7}$

To understand the problem posed by the development of dynamical spacetime theories, it will be worthwhile to take a closer look at how the isometries of a spacetime are defined. The initial concern is that the comparison of any geometrical object, such as the metric, at different points requires a definition of distance, and it is the metric itself that provides the definition of distance. Certainly, we cannot use the metrical properties of the space to study the metrical properties of the spacethis would be the equivalent of using a meter stick to measure how meter sticks change from place to place. ${ }^{8}$ Rather, we need a way to characterize the change in the metric brought about by a motion in the manifold - that is, we need a way to study the changes in the metrical properties of a space that does not implicitly rely on the metric itself.

This is where the concepts of Lie dragging and the Lie derivative come in handy because they can be defined on a manifold without a metrical structure. The basic idea behind the concept of Lie dragging is quite simple. We can fill a manifold with a congruence of curves-that is, a collection of nonintersecting curves. In this case, each point in the manifold will lie on one, and only one, curve in the congruence. The congruence of curves provides a natural way to characterize a mapping of the manifold onto itself: if the parameter on each curve is $\lambda$, then $\Delta \lambda$-that is, some small number-defines a mapping in which each point is mapped to one a parameter distance $\Delta \lambda$ farther along the same curve (e.g., see Schutz 1980, 73). Such a mapping is called a Lie dragging, and a quantity is said to be Lie dragged if it is left unchanged by the transformation. Of course, we can Lie drag geometrical objects, such as the metric - formally, the metric tensor field — defined on the manifold to study their properties as they are "dragged" across the manifold.

The concept of Lie dragging also allows us to define a derivative along the congruence of curves. The typical derivative takes the difference of a scalar or tensor defined at different points divided by the distance between them. However, without a metric, we cannot provide a definition of this distance between noncoincident points.

\footnotetext{
${ }^{6}$ Noether's theorem has been addressed at length in the philosophical literature (see, for example, Brading and Brown [2003]; Earman [2003]; Brown and Holland [2004]; Butterfield [2005]).

${ }^{7}$ Imagine trying to define a conservation law in a space with an arbitrary metrical structure that changes from point to point. What would energy or momentum even mean in such a space?

${ }^{8}$ Any change in the length of the meter stick under study would be imperceptible because of the equivalent change in the measuring meter stick.
} 
Furthermore, without a connection, the space also lacks a definition of parallelism, and we cannot fix the concept of motion in a set direction. ${ }^{9}$ In fact, without reference to a connection, the question of parallelism makes little to no sense. Thankfully, the congruence of curves itself can serve as a substitute for the concept of parallelism at different points. We can consider points along the same curve to be "parallel" and compare the metric evaluated at points $\lambda$ and $\lambda+\Delta \lambda$ along the curve. If we Lie drag the metric evaluated at $\lambda+\Delta \lambda$ back to $\lambda$, and cancel any effect of the inherent coordinate transformation, we can compare the two values to define a unique derivative, known as the Lie derivative. If the Lie derivative of the metric tensor is zero along a particular congruence of curves, then we say that the metric tensor is invariant under translation along that congruence. Of course, a congruence of curves also defines a vector field-namely, the field of tangents to the curves. If the metric tensor is invariant with respect to a given congruence and its associated vector field, then we call it a Killing vector field. The Killing vector fields define the isometries of the manifold.

We can now use these structures to study the conservation laws in a dynamical spacetime theory. At the outset, it is clear that we can only derive a global conservation law in spacetime structures with the relevant Killing vector fields. This is not typically a problem in classical mechanics or quantum theory because the relevant spacetime structures are maximally symmetric and typically possess the required Killing vector fields to secure the conservation of energy-momentum. ${ }^{10}$ However, an arbitrary dynamical spacetime will not possess any Killing vector fields and, therefore, no continuous isometries (e.g., see Hawking and Ellis 1973, 44). In an arbitrary dynamical spacetime, we cannot define any globally conserved quantities or global conservation laws. ${ }^{11}$

All of this may not be a serious concern so long as we can show that the dynamical spacetime structure of general relativity has the relevant Killing vector fields. The problem is that the imposition of a set of continuous isometries is, in general, a violation of the fundamental principles of general relativity. We know that general relativity, strictly speaking, forbids the imposition of this type of symmetry because the imposition of a Killing vector field is acausal-it requires imposing a structure on regions of spacetime that are outside our light cone. ${ }^{12}$ We have no causal connection and no epistemic access to these regions of spacetime. Furthermore, there is no reason to suspect that these regions of spacetime possess the requisite symmetry because we do not even believe that the observable regions of spacetime do. Fundamentally, we know that no symmetric stress-energy tensor could accurately describe the local arrangement of energy and matter in the neighborhood of

\footnotetext{
${ }^{9}$ A connection provides a means of relating the tangent spaces of nearby points and defines the notion of parallel transfer.

${ }^{10}$ For example, the Minkowski spacetime of special relativity and quantum field theory has ten Killing vector fields-four translational and six rotational (three corresponding to the spatial rotations and three to the special Lorentz transformations).

${ }^{11}$ There may exist conservation laws that are not associated with a Killing vector but rather with a nontrivial Killing tensor. However, more work still needs to be done to understand the nature of these conservation laws.

${ }^{12}$ This is because the Killing vector fields are defined by acausal elliptical partial differential equations.
} 
Earth, simply because the local arrangement of matter and energy in the observable universe is not symmetric. Strictly speaking, it is also unlikely that any global symmetries exist either-even the microwave background radiation exhibits variation. It seems that any realistic local spacetime structure in general relativity will not possess any Killing vector fields or global conservation laws. ${ }^{13}$

However, despite these concerns, we can still secure a local account of the conservation of energy, at least as an approximation, within the shared geometricalmathematical framework of modern physics. This shared framework-whereby all spacetime structures are taken to constitute a differentiable Riemannian, or pseudo-Riemannian, manifold-allows for the local specification of the typical Lie symmetry groups. The regular nature of the Riemannian spacetimes of modern physics ensures that the typical conservation laws apply "locally" in the neighborhood of all points in a spacetime, at least approximately, and that these symmetries will hold on the global scale in a certain special class of spacetime structures. But can we give a viable perspectival account of this notion of retention and continued success?

It is only from the perspective of modern differential geometry that we can define the sense in which the laws of conservation of energy found in static spacetime theories are retained as local laws in a dynamical spacetime theory, such as general relativity. In a neo-Kantian account, we could simply suggest that this geometricalmathematical framework serves as a constitutive framework for modern physics and secures the objectivity of the law of conservation of energy. However, in a perspectival account, it is not immediately clear what role we should assign to this mathematical framework.

There is no question that the shared geometrical-mathematical formalism of modern physics can secure the interperspective translatability required to ground Massimi's account of perspectival truth. The geometrical structure of static spacetime theories could be viewed as a particular instance of a more general dynamical spacetime theory. But we simply cannot define the empirical testability, projectability, fruitfulness, and so forth of the law of conservation of energy, at least from the perspective of general relativity, without this mathematical formalism. To defend a viable perspectivism in modern physics, something must be said about how its mathematical methodology can secure a genuine correspondence claim.

\section{The dilemma of perspective}

In response to this challenge, Massimi's perspectivist is unable to simply argue that the mathematical formalism of modern physics represents features of an objective

\footnotetext{
${ }^{13}$ In response, some might note that the big bang Friedmann-Lemaître-Robertson-Walker (FLRW) spacetime of general relativity is highly symmetric and certainly allows for conservation laws. But the FLRW solution is spatially homogeneous and isotropic, and it is based on the assumption that there exists a family of nonintersecting, space-like, maximally symmetric hypersurfaces that fill the spacetime. The imposition of this structure is a result of the fact that we are generally only able to provide exact solutions for the Einstein field equations in highly symmetric spacetime structures. And just because these are typically the only solutions that we can obtain, this does not mean that the theory itself condones the imposition of this type of symmetry.
} 
mind-independent reality because they deny the realist appeal to objectivity. Instead, they may wish to argue that this framework is itself merely a broader perspective from which we conduct scientific inquiry. However, in this case, the perspectival account of truth would be indexed to this perspective. The retention and continued success of scientific claims through the progress of science is then only definable within a scientific perspective. This would clearly undermine any claim that the perspectivist could make to defending a form of realism.

If the perspectival realist cannot appeal to a direct conformity between mathematics and nature or to a broader mathematical perspective of physical inquiry, maybe they could try to find a viable middle ground. The perspectivist might try to defend a sort of meta-perspectival argument that suggests that the mathematical framework of modern physics is of more than a mere instrumental value. Although it may not directly represent the structure of the natural world, the relevant mathematical framework, or some further generalization thereof, may be taken to be an essential feature of the practice of modern physics. Certainly, we could appeal to inductive support for this claim.

The problem is to precisely define the sense in which this framework is essential to scientific practice, beyond its instrumental value. Once again, we are confronted with the problem of the applicability of mathematics. It is not enough to claim that a given mathematical framework has been successful in the description of a given domain. This would lead as much to instrumentalism as realism. Rather, the perspectivist needs to clarify what it is about a given mathematical formalism that allows it to support the claim that the facts we hold to be true are in some sense facts about a nature that is independent of our representations.

It would certainly not be controversial to put forward a defense of the privileged role of mathematics in physical inquiry. The Scientific Revolution was based, in part, on a mathematization of nature. Galileo $(1623 / 1957,238)$ famously wrote that the book of nature "is written in the language of mathematics," without which it is "impossible to understand a single word of it." In the context of modern theoretical physics, it seems that Galileo's mathematization of nature has reached its purest expression. It would not be unreasonable to suggest that this mathematization of nature will persist through the perspectival series of scientific theories. Therefore, the perspectivist could suggest that the mathematical methodology of modern physics is truth-conducive in the sense that the shared mathematical formalism of physics could provide a unified framework through which the structural features of a scientific perspective that are retained through the perspectival series of scientific theories can be said to have some grip on reality.

This sort of epistemological Pythagoreanism should be distinguished from the popular structural realist position (e.g., see Worral 1989; Ladyman 1998; Ladyman and Ross 2007; French 2014). Structural realism can be understood in its epistemic variant as a simple limitation on scientific knowledge or, in its ontological variant, as a claim about the metaphysics of science. However, in either case, the structural realist seeks to maintain the typical realist claim of objectivity in the face of underdetermination and discontinuity in the history of science. Perspectivism abandons the objectivity of scientific claims in favor of a minimal notion of scientific truth established as a form of resilience and coherence across the progress of science. The retained claims in modern physics (e.g., local conservation laws) can be 
understood in perspectival terms to be true-but not as an objective, God's-eye view, fact about nature. Thus, the knowledge that underwrites this form of epistemological Pythagoreanism would remain perspectival knowledge.

The dilemma is that mathematics is a human pursuit, in much the same way that science is, and there seems to be no reason to suspect that this human pursuit would somehow provide the key to understanding nature (e.g., see Steiner 1998) —at least from a perspectival point of view. Arguing that one can secure the truth of successful scientific claims from the perspective of a given mathematical framework does not lead to realism, at least if this framework itself is taken to be no more than a perspective. As Cassirer might argue, the very thing that the perspectivist seems to need to secure a genuine realism appears to be that which they cannot give. They must privilege a perspective-for example, the mathematical framework of differential geometry.

The problem is not that such a view would be difficult to defend. As Dirac $(1939,1)$ famously wrote:

There is no logical reason why the [method of mathematical reasoning in physics] should be possible at all, but one has found in practice that it does work and meets with reasonable success. This must be ascribed to some mathematical quality in Nature, a quality which the casual observer of Nature would not suspect, but which nevertheless plays an important role in Nature's scheme. ${ }^{14}$

Similarly, Feynman $(1964,59)$ writes:

When learning the Laws of Physics you find that there are a large number of complicated and detailed laws, laws of gravitation, of electricity and magnetism, nuclear interactions, and so on, but across the variety of these detailed laws there sweep great general principles which all the laws seem to follow. Examples of these are the principles of conservation, certain qualities of symmetry, the general form of quantum mechanical principles, and unhappily, or happily, ... the fact that the laws are mathematical. ${ }^{15}$

The idea that the underlying mathematical framework of modern physics might serve as a general principle from which all natural law is derived can also be found in the writings of a number of other prominent physicists - for example, Gell-Mann (1994) and Penrose (2004). On the support of authority alone, it seems that we are in good company in highlighting the mathematical framework that underwrites appeals to natural law.

The problem is that in defending such a view, we run the risk of undermining the original motivation of scientific perspectivism. To privilege mathematics would be to deny the perspectivist dogma and would threaten to collapse perspectivism into some undesirable form of neo-Pythagoreanism. The perspectivist could simply return to the central Kantian dogma that all knowledge is from a human vantage point, but if the methodology underwriting this knowledge is itself just another vantage point,

\footnotetext{
${ }^{14}$ Emphasis added.

${ }^{15}$ Emphasis added.
} 
then in what sense can the perspectivist hope to defend any form of scientific realism?

Indeed, this concern seems to present a challenge to the viability of perspectivism more generally. Here, it is important to note that it is not Massimi's perspectivism that is the problem, per se, but rather the correspondence theory of truth that she seeks to defend. To ensure that the claims that we hold to be true are consistent and can be taken to correspond to a unique mind-independent reality, we are required to impose a strict form of continuity and coherence across the progress of science. Thus, any form of perspectivism that looks to defend a correspondence theory of truth will have to face a similar challenge in providing a realist account of the applicability of mathematics in the methodology of physical inquiry.

In response, some might wonder whether the solution may be to simply abandon the correspondence theory in favor of a more liberal pragmatic theory of truth (e.g., Teller 2012, 264-66). In Teller's $(2012,2019)$ pragmatic perspectivism, a scientific claim is said to be true if it works, at least well enough, in the intended domain of application. To "work" is to satisfy one's aims in a given scientific context. These aims should not be understood to be indexed to an individual but rather to the scientific community as a whole. The truth of a scientific claim is defined by its success in a broad range of applications (Teller 2019, 59). It is in this sense that Teller (2012, 272) holds that even though all science is idealized and imprecise, one can still argue that it gives us a "real cognitive grip on real things."

Adopting a pragmatic theory of truth would allow us to sidestep the issue of securing a clear form of resilience and coherence of scientific claims across the progress of science because all that matters is whether the claims work in their intended domains of application. Teller's $(2011,2012,2019)$ perspectivism offers an even more humble picture of scientific realism. His realist commitment is limited to those claims that function in the appropriate sense in a given context. But unless the domain of application is broad enough to include multiple scientific perspectives, a pragmatic perspectivism would remain far too local to constitute a viable scientific realism. It would only be able to support a notion of scientific truth within the limits afforded by a given scientific theory. Most realists would hope for more. Even in a pragmatic theory, we would need to appeal to the broader methodology of science to secure a sense in which perspectival claims can transcend the bounds of a single perspective.

It is unclear if a pragmatic perspectivism will fare any better in addressing the applicability of mathematics in modern physics. To define the sense in which a given scientific claim (e.g., that energy is conserved) "works" across a broad range of applications (e.g., in static and dynamic spacetime theories), the pragmatist must still appeal to the formalism of modern differential geometry to define the manner in which the laws of conservation of energy found in static spacetime theories are retained as local laws in a dynamical spacetime theory. It is this framework that defines what the conservation of energy means in a given domain. Outside this framework, the pragmatic perspectival realist would not even be able to define what it means for the claim that energy is conserved to "work" in a dynamical spacetime. Making matters worse, Teller's more permissive perspectivism may be unable to give any account of truth concerning the broader mathematical methodology of modern 
physics, which defines what it means for a claim to "work" in this context. ${ }^{16}$ Thus, the neo-Kantian critique concerning the applicability of mathematics in physics may pose a more general challenge to scientific perspectivism, as a whole.

\section{Conclusion}

In this article, I have argued that there is an inherent tension in the perspectival appeal to the methodology of science to secure an account of scientific knowledge. In the context of modern physics, the perspectival realist has to either privilege a given mathematical framework or abandon the aims of perspectivism. The methodology of science can serve to define the manner in which perspectival claims can transcend the bounds of mere perspective. However, it is unclear how perspectivists can privilege a methodology without abandoning the central tenet of perspectivism.

Perspectival realism offers a refreshing new perspective on the realism debateone that is more in line with the actual practice of modern science. The perspectivist provides a clear account of how we can maintain a form of realism despite the disparate nature of modern scientific inquiry. Although I have argued that the perspectivist may be unable to account for the methodology of modern mathematical physics, this does not mean that the account cannot be consistently applied in other domains (e.g., see Massimi and McCoy 2019). However, the concerns that I have addressed in this article may not be limited to mathematical physics alone. Recently, Chirimuuta (2019) has argued that in neuroscience, one often finds theories that share only a mathematical continuity. In this case, the only form of perspectivism that may be tenable is one that is grounded on a particular mathematical framework. But as Chirimuuta $(2019,152)$ has shown, this is problematic, given that the relevant mathematics is subject to radically divergent interpretations. Thus, it seems that the problem of the applicability of mathematics may pose a broad concern for the perspectivist. Given the increasingly mathematical nature of modern science, the problems addressed in this article may extend beyond the field of mathematical physics and threaten the viability of scientific perspectivism more generally.

Acknowledgments. I would like to thank Jennifer Fraser, Joseph Berkovitz, James Robert Brown, Paul Teller, Michela Massimi, Ana-Maria Cretu, and the audience at the 2021 Alternative Approaches to Scientific Realism Conference at the Munch Center for Mathematical Philosophy for helpful comments. I also want to thank the Azrieli Foundation and the Dan David Foundation for generously supporting this research.

\section{References}

Brading, Katherine, and Harvey Brown. 2003. "Symmetries and Noether's Theorems." In Symmetries in Physics: Philosophical Reflections, edited by Katherine Brading and Elena Castellani, 89-109. Cambridge: Cambridge University Press.

Brown, Harvey, and Peter Holland. 2004. "Dynamical versus Variational Symmetries: Understanding Noether's First Theorem." Molecular Physics 102 (11-12):1133-39.

\footnotetext{
${ }^{16}$ However, as Teller $(2012,266)$ notes, his pragmatic perspectivism is still an open avenue of research, and more work still needs to be done to determine the exact form of realism it licenses.
} 
Butterfield, Jeremy. 2005. "On Symmetry and Conserved Quantities in Classical Mechanics." In Physical Theory and Its Interpretation: Essays in Honor of Jeffrey Bub, edited by William Demopoulos and Itamar Pitowsky, 89-109. Dordrecht: Springer.

Carnap, Rudolf. 1956. Meaning and Necessity: A Study in Semantics and Modal Logic. Chicago: University of Chicago Press.

Cassirer, Ernst. 1910/1923. Substance and Function. Reprint, New York: Dover Publications.

Cassirer, Ernst. 1925/1946. Language and Myth. Reprint, New York: Dover Publications.

Cassirer, Ernst. 1936/1956. Determinism and Indeterminism in Modern Physics. New Haven: Yale University Press.

Chakravartty, Anjan. 2007. A Metaphysics for Scientific Realism: Knowing the Unobservable. Cambridge: Cambridge University Press.

Chirimuuta, M. 2019. "Charting the Heraclitean Brain: Perspectivism and Simplification in Models of the Motor Cortex." In Understanding Perspectivism: Scientific Challenges and Methodological Prospects, edited by Michela Massimi and Casey McCoy, 141-59. New York: Routledge.

Dirac, Paul. 1939. "The Relation between Mathematics and Physics." Proceedings of the Royal Society (Edinburgh) 59 (2):122-29.

Earman, John. 2003. "Laws, Symmetry, and Symmetry Breaking: Invariance, Conservation, and Objectivity." Philosophy of Science 71 (5):1227-41.

Feynman, Richard. 1964. The Character of Physical Law. Cambridge: MIT Press.

French, Steven. 2014. The Structure of the World: Metaphysics and Representation. Oxford: Oxford University Press.

Galileo Galilei. 1623/1957. "Opere Il Saggiatore." In Discoveries and Opinions of Galileo, translated by Stillman Drake, 229-80. Reprint, New York: Doubleday and Company.

Gell-Mann, Murray. 1994. The Quark and the Jaguar: Adventures in the Simple and the Complex. New York: Freeman.

Giere, Ronald. 2006. Scientific Perspectivism. Chicago: University of Chicago Press.

Goldstein, Herbert. 1980. Classical Mechanics. Reading: Addison-Wiley.

Goodman, Nelson. 1976. Languages of Art. Indianapolis: Hackett Publishing Company.

Goodman, Nelson. 1978. Ways of Worldmaking. Cambridge: Hackett Publishing Company.

Hawking, Stephen, and George Ellis. 1973. The Large Scale Structure of Space-Time. Cambridge: Cambridge University Press.

Kuhn, Thomas. 1962. The Structure of Scientific Revolutions. Chicago: University of Chicago Press.

Ladyman, James. 1998. "What Is Structural Realism?" Studies in the History and Philosophy of Science 29 (3):409-24.

Ladyman, James, and Don Ross. 2007. Everything Must Go. Oxford: Oxford University Press.

Massimi, Michela. 2012. "Scientific Perspectivism and Its Foes." Philosophica 84 (1):25-52.

Massimi, Michela. 2016. "Four Kinds of Perspectival Truth." Philosophy and Phenomenological Research 96 (2):342-59.

Massimi, Michela. 2018a. "Perspectival Realism." In The Routledge Handbook of Scientific Realism, edited by Juha Saatsi, 164-75. New York: Routledge.

Massimi, Michela. 2018b. "A Perspectivalist Better Best Systems Account of Lawhood." In Laws of Nature, edited by Walter Ott and Lydia Patton, 139-57. Oxford: Oxford University Press.

Massimi, Michela. 2018c. "Perspectival Modeling." Philosophy of Science 85 (3):335-59.

Massimi, Michela, and Casey McCoy. 2019. Understanding Perspectivism: Scientific Challenges and Methodological Prospects. New York: Routledge.

Morrison, Margaret. 2011. "One Phenomenon, Many Models: Inconsistency and Complementarity." Studies in History and Philosophy of Science 42 (2):342-51.

Nietzsche, Friedrich. 1873/2010. On Truths and Lies in a Non-Moral Sense. Translated by Taylor Carmen. Reprint, New York: Harper Perennial Modern Thought.

Penrose, Roger. 2004. The Road to Reality: A Complete Guide to the Laws of the Universe. London: Vintage.

Putnam, Hilary. 1981. Reason, Truth and History. Cambridge: Cambridge University Press.

Putnam, Hilary. 1987. The Many Faces of Realism. LaSalle: Open Court.

Rueger, Alexander. 2005. "Perspectival Models and Theory Unification." British Journal for the Philosophy of Science 56 (3):579-94.

Rueger, Alexander. 2016. “Perspectival Realism and Incompatible Models.” Axiomathes 26 (4):401-10. 
Schutz, Bernard. 1980. The Geometrical Methods of Mathematical Physics. Cambridge: Cambridge University Press.

Steiner, Mark. 1998. The Applicability of Mathematics as a Philosophical Problem. Cambridge: Harvard University Press.

Teller, Paul. 2011. "Two Models of Truth.” Analysis 713 (3):465-72.

Teller, Paul. 2012. "Modelling, Truth, and Philosophy." Metaphilosophy 43 (3):257-74.

Teller, Paul. 2019. "What Is Perspectivism, and Does It Count as Realism?" In Understanding Perspectivism: Scientific Challenges and Methodological Prospects, edited by Michela Massimi and Casey McCoy, 49-64. New York: Routledge.

Weyl, Herman. 1952. Symmetry. Princeton: Princeton University Press.

Worrall, John. 1989. "Structural Realism: The Best of Both Worlds?” Dialectica 43 (1-1):100-24.

Cite this article: Stemeroff, Noah. 2022. "Scientific Perspectivism and the Methodology of Modern Mathematical Physics." Philosophy of Science 89 (3):504-520. https://doi.org/10.1017/psa.2021.34 